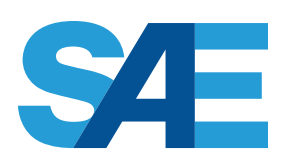

INTERNATIONAL

\title{
Development of Bicycle Surrogate for Bicyclist Pre-Collision System Evaluation
}

\section{Qiang Yi, Stanley Chien, Jason Brink, Wensen Niu, Lingxi Li, and Yaobin Chen}

Indiana University Purdue University

\section{Chi-Chen Chen}

Ohio State University

\section{Rini Sherony \\ TEMA}

\section{Hiroyuki Takahashi}

Toyota Motor Corporation

CITATION: Yi, Q., Chien, S., Brink, J., Niu, W. et al., "Development of Bicycle Surrogate for Bicyclist Pre-Collision System Evaluation," SAE Technical Paper 2016-01-1447, 2016, doi:10.4271/2016-01-1447.

\begin{abstract}
As part of active safety systems for reducing bicyclist fatalities and injuries, Bicyclist Pre-Collision System (BPCS), also known as Bicyclist Autonomous Emergency Braking System, is being studied currently by several vehicles manufactures. This paper describes the development of a surrogate bicyclist which includes a surrogate bicycle and a surrogate bicycle rider to support the development and evaluation of BPCS. The surrogate bicycle is designed to represent the visual and radar characteristics of real bicyclists in the United States. The size of bicycle surrogate mimics the 26 inch adult bicycle, which is the most popular adult bicycle sold in the US. The radar cross section (RCS) of the surrogate bicycle is designed based on RCS measurement of the real adult sized bicycles. The surrogate bicycle is constructed with detachable components with shatter resistant material to prevent structural damage during a collision, and matches the look and RCS of a real 26 inch mountain bicycle from all 360 degree angles. The surrogate bicycle rider is a $168 \mathrm{~cm}$ tall adult with CNC machined realistic body shape. The skin of the surrogate bicycle rider has the RCS of a real human skin. Combined skin with realistic body shape, the surrogate bicyclist has the RCS matching to that of a same sized real human from 360 degree angles in the view of $77 \mathrm{GHz}$ automotive radar. The surrogate bicyclist has articulated leg motion which is important for micro Doppler sensing and can be supported on a sled or a mobile carrier. It can be moved at a speed of $20 \mathrm{mph}$ and can be collided by vehicles from any direction and be reassembled in less than 5 minutes.
\end{abstract}

\section{Introduction}

According to the crash data of the U.S. Fatality Analysis Report System (FARS), 743 pedal cyclists were killed and an estimated 48,000 pedal cyclists were injured in motor vehicle traffic crashes in 2013 [1]. Pedal cyclist deaths accounted for 2 percent of all motor vehicle traffic fatalities and injured pedal cyclists made up 2 percent of the people injured in traffic crashes during the year. The number of the pedalcyclists killed in 2013 is 1 percent higher than the 734 pedal cyclists killed in 2012. The increase in 2013 is the third straight increase in pedal cyclist fatalities, a 19-percent increase since 2010 [1].

As a novel active safety equipment to reduce pedalcyclist injuries and fatalities, Bicyclist Pre-Collision System (BPCS), also referred as bicyclist collision imminent breaking system (CIB) or bicyclist automatic emergency braking (AEB), have been studied and introduced by several vehicle manufacturers in recent years[2 $\underline{2}, \underline{3}]$. BPCS automatically generates braking when there is an imminent frontal collision with a bicyclist and the driver does not apply braking. BPCS is an added feature of the AEB systems and operates similar to AEB now being implemented for vehicles and pedestrians.

To support the standardized evaluation of the BPCS, the test scenarios, test equipment and test procedures for BPCS has been studied by Transportation Active Safety Institute (TASI) at Indiana University-Purdue University Indianapolis (IUPUI) under the support of Collaborative Safety Research Center (CSRC) of Toyota. This

This is the author's manuscript of the article published in final edited form as: Yi, Q., Chien, S., Brink, J., Niu, W., Li, L., Chen, Y., ... Takahashi, H. (2016). Development of Bicycle Surrogate for Bicyclist Pre-Collision System Evaluation. https://doi.org/10.4271/2016-01-1447 
paper describes the development the surrogate bicyclist. One of the requirements is to develop a bicycle surrogate that is independent of any specific BPCS implementation, but relevant to the current and foreseeable future BPCS technology. Therefore, the surrogate bicyclist target should have similar physical properties as a representative bicyclist with respect to most common sensors used in vehicles for bicyclist detection in all viewing angles. References $[\underline{4}, \underline{5}, \underline{6}]$ show that cameras and radar are main sensors used for detecting bicyclists. Thus, the designed surrogate bicyclist for BPCS evaluation should have the same visual and radar characteristics of real bicyclists. In addition, other important requirements for bicycle surrogate include surviving from repeated crashes, having low weight for test vehicle safety and having fast recovery time.

\section{Type and Size of the Surrogate Bicycle}

The representative type and size of surrogate bicyclists can provide a realistic evaluation of BPCS. To determine the type and size of surrogate bicycles, two important investigations were conducted; they are bicycle market investigation and bicyclist fatalities and injury analysis.

\section{Investigation of Bicycle Consumption}

Because most bicycles sold in the US are imported, a summary of recent years' import bicycles with different wheel size and type were obtained from 2012 Bicycle Product Supplier Association (BPSA) Statistical Report. Figure 1 and Table 1 are created based on the information in the report. Figure 1 tells that the wheel sizes of majority of bicycles imported to U.S. market were 19 or 20 inch wheels for kid's bicycles and 26 inch wheels for adult bicycles. Table 1 shows the frame types of majority adult bicycles are mountain bicycles and road bicycles.

Therefore, we decide that the wheel size of the adult surrogate bicycle should be 26 inches and that of the child surrogate bicycle should be 19 or 20 inches. The type of adult surrogate bicycle is defined as a mixed shape of mountain and road type.

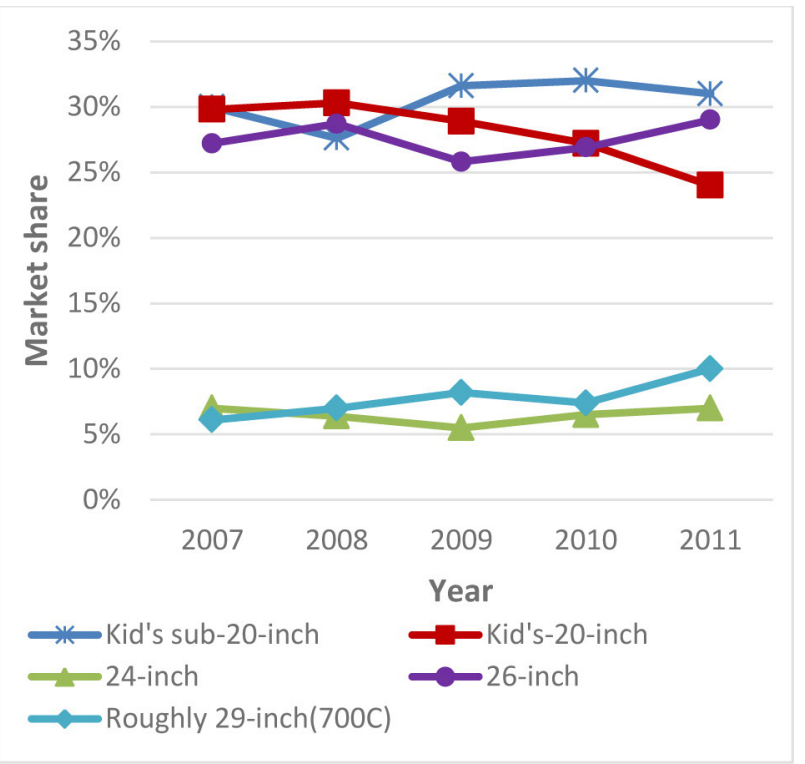

Figure 1. Bicycle import unit market by category. Source: U.S. Department of Commerce.
Table 1. Bike shop 2010 and 2011 unit consumption by product category.

\begin{tabular}{|l|r|r|}
\hline Frame Style & 2010 percent & 2011 percent \\
\hline Adult - Mountain & 27.00 & 28.30 \\
\hline Adult - Road and racing bike & 17.10 & 17.40 \\
\hline $\begin{array}{l}\text { Adult - Hybrid/Cross (blend of } \\
\text { road, mountain, and touring) }\end{array}$ & 21.60 & 22.70 \\
\hline Youth (16"-24" wheel) & 21.10 & 20.90 \\
\hline Other & 13.20 & 10.70 \\
\hline Total & 100.00 & 100.00 \\
\hline
\end{tabular}

\section{Bicyclist Fatalities and Injuries by Age and Gender}

According to "Bicycling and walking in the United States, 2014 benchmarking report," [7] adults between age 16 to age 64 share $77 \%$ and $68 \%$ of the whole bicyclist fatalities and injuries, respectively. Seniors aged 65 or older represented $12 \%$ of all bicyclist fatalities and $5 \%$ of all bicyclist injuries. Children under age 16 represented $11 \%$ of total bicyclist fatalities and $27 \%$ of total bicyclist injuries between 2009 and 2011 (see Figure 2). Since the bicyclists over age 16 covered $89 \%$ of fatalities and $95 \%$ of injuries, and some teenaged children were also adult sized, only the adult surrogate bicyclist size was considered.

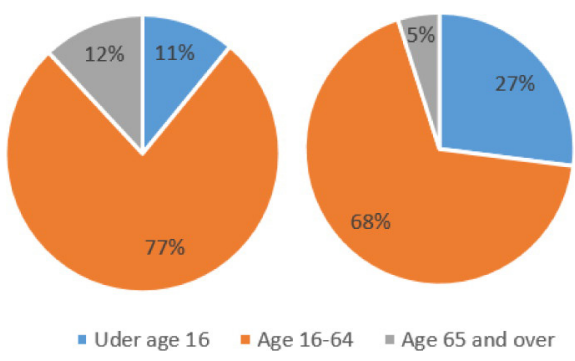

Figure 2. Bicyclist Fatalities (left) and Injuries (right) by Age from year 2009 to year 2011(source: [7]).

Figure 3 depicts charts of bicyclist fatalities and injuries in 2013. These charts show that the majority of the bicyclists killed and injured were male, representing $87 \%$ of fatalities and $83 \%$ of injuries, respectively. Considering average heights of the male and the female over 13 years old in the US, and weighted them based on the percentage of gender shown in Figure 2, the representative height of the surrogate bicycle rider in the US should be $171 \mathrm{~cm}$.

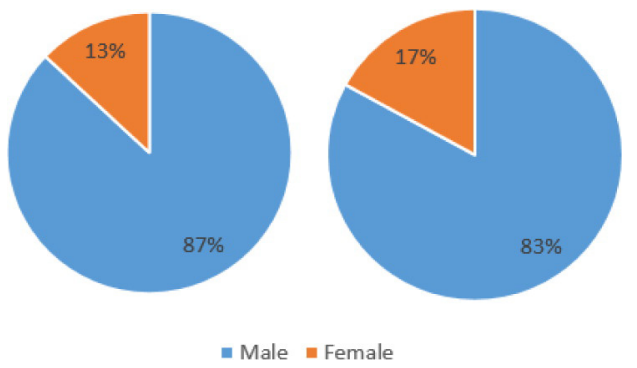

Figure 3. Bicyclist Fatalities (left) and Injuries (right) by Gender in 2013 (source: [7]).

The adult fit mannequin for pedestrian pre-collision system evaluation has been modified as a surrogate bicycle rider. All shoulder, hip, and knee joints are passively rotatable (no actuator on 
these joints). There are no elbow joints. The skin of the surrogate bicyclist has the RCS of a real human skin. The realistic body shape of mannequin is CNC (Computer Numerical Control) machined (see Figure 4) in order to generate proper the radar cross section (RCS) of corresponding sized human being from a 360 degree angle in the view of $77 \mathrm{GHz}$ automotive radar. More details about the mannequin development can be found in []]

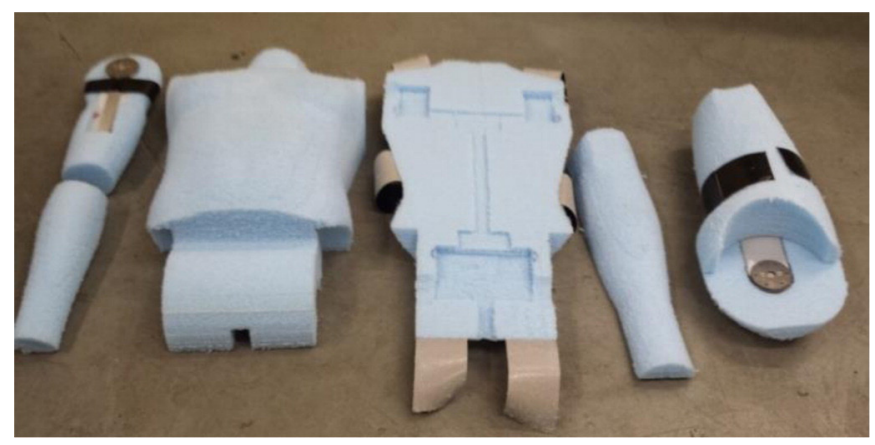

Figure 4. CNC machined adult mannequin foam pad parts.

\section{Wheel Design of the Surrogate Bicycle}

Based on the RCS measurement of 10 bicyclists, it was found that the main contributor of the bicycle RCS was from the rider when viewed from front and rear, and was from the bicycle wheel when viewed from the sides. Based on the RCS measurements of real bicycle wheels (shown in Figure 5), the RCS of the rubber tire was negligible comparing to that of the rim and spokes. So the RCS part of the wheel design was focused on the rim and spokes. To ensure that the wheels are not damaged during the crash, the surrogate bicycle wheel needs to be able to deform and return to original shape after the crash. Therefore, a flexible and transparent printed circuit board (PCB) with copper or silver traces was used to emulate the real rim and spokes. The detail design is shown in Figure 6. This flexible PCB film is sandwiched by two 25.25 inches $(641 \mathrm{~mm})$ diameter $0.8 \mathrm{~mm}$ thick polycarbonate disks. The edge of the sandwiched disk is optionally covered by polyethylene foam tube (as the inner tube of the bike wheel) and then covered by a real 26 inch bicycle rubber tire. The resulting wheel has the look of a bike wheel, but can be bent and crashed (shown in Figure 7).

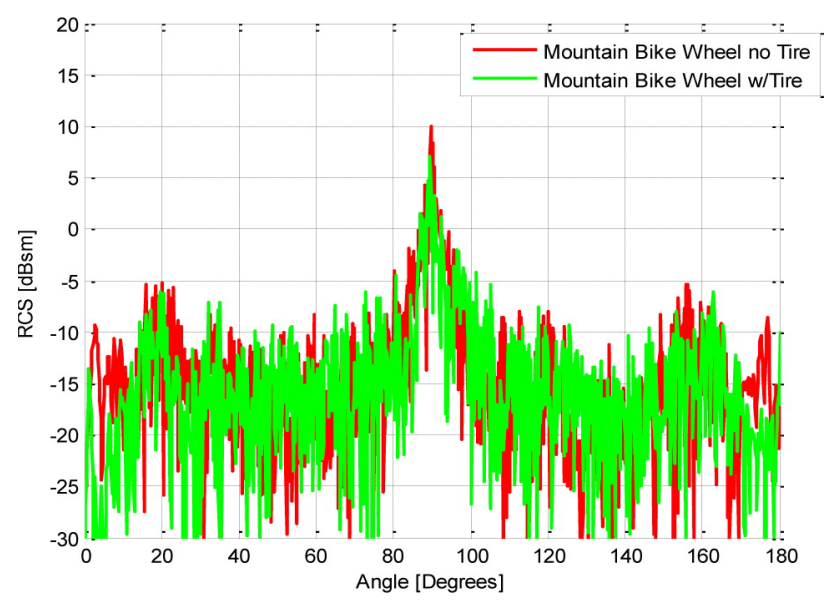

Figure 5. The RCS of the bicycle tire.
Figure 8 shows that the comparison between the smoothed RCS of the design of the rim and spokes and the RCS of the implemented $\mathrm{PCB}$ rim and spokes (smoothed by 2 degree window). Zero degree is the front view (facing the face), 180 degrees is the back view and 90 degrees is the side view. It demonstrates that the RCS of the PCB emulated combined surrogate bicycle rim and spokes matches with the RCS of the actual combined bike rim and spokes from 65 to 105 degrees. Although the RCS of the combined rim and spokes of a surrogate wheel and the RCS of a real wheel do not match well beyond this range, the overall RCS of the surrogate bicyclist is not affected since the RCS contribution of wheels to that of the whole real bicyclist is small beyond 65 to 105 degrees anyway.

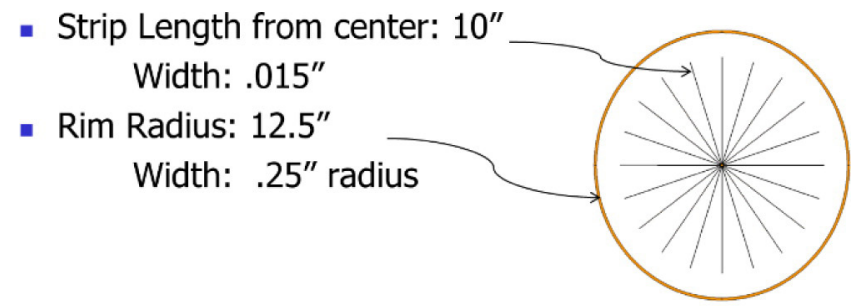

Figure 6. Wheel flexible PCB model.

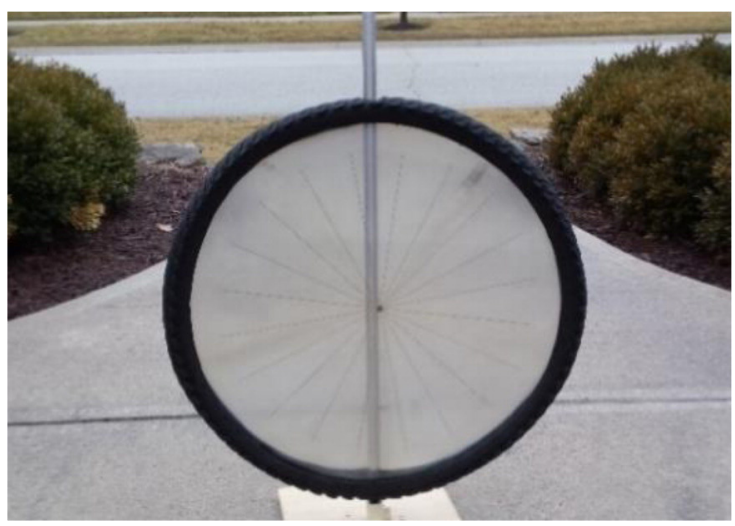

Figure 7. Surrogate wheel construction.

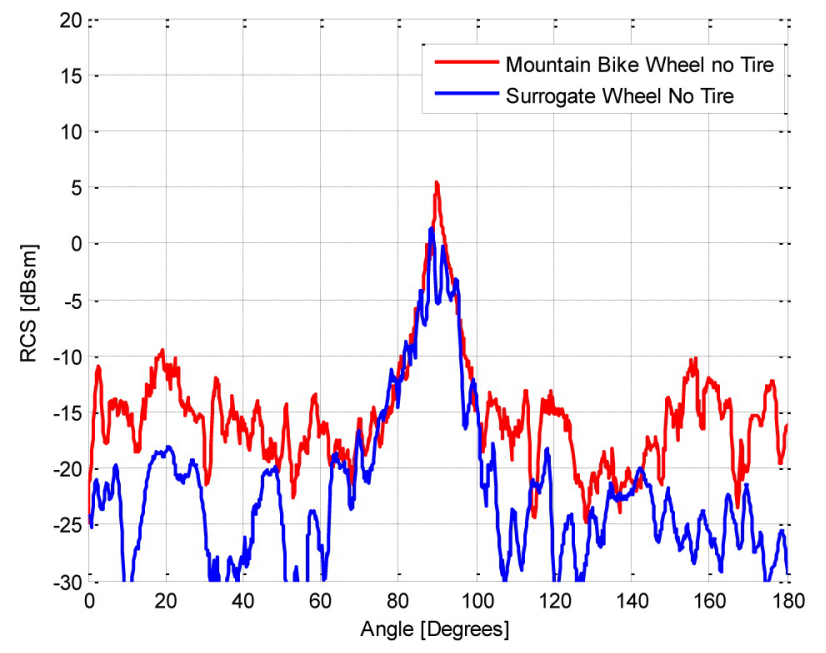

Figure 8. The comparison between the RCS of the real rim and spokes to the implemented PCB rim and spokes (2 degree window smoothed). 


\section{Frame Design of the Surrogate Bicycle}

The surrogate bicycle frame is used to support the bicycle rider and hold wheels. It consists of six main parts: seat tube, top tube, down tube connection tube, handle bar and head tube (shown in Figure 9). To reduce potential damage in crash testing and recovery time after crash, bungee cords are used to connect all frame parts by running through the frame pieces and be knotted at connection points (see Figure 10). The surrogate bicycle frame is completely connected due to the compressive force from the bungee cords. When the vehicle crashes into the surrogate, the frame pieces physically come apart at the joints momentarily but still connected by the bungee cords. All components of the surrogate bicycle frame can be realigned to original shape by hands in seconds. To generate the same level of RCS as in a real bicycle frame, all frame parts are covered by a layer of copper fabric for emulating metal surface. A layer of foam is inserted between the copper fabric and frame tube to achieve required thickness and protect the bike and vehicle during crash.

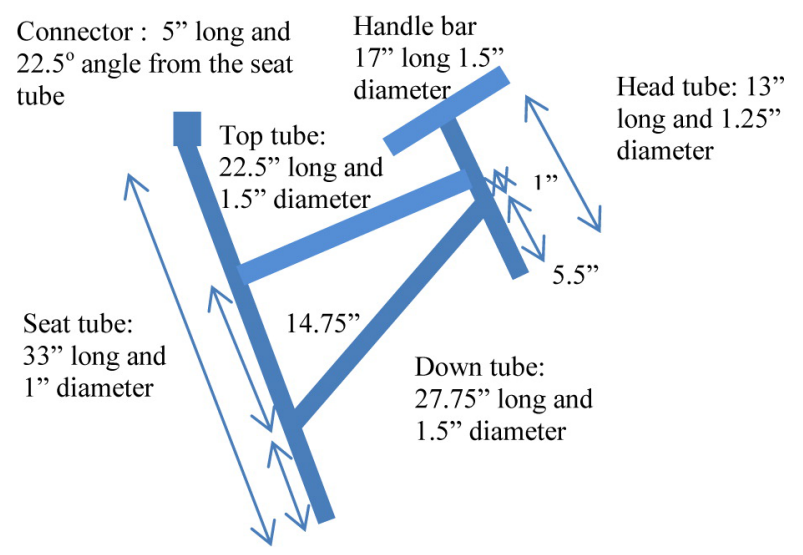

Figure 9. Bicycle frame parts.

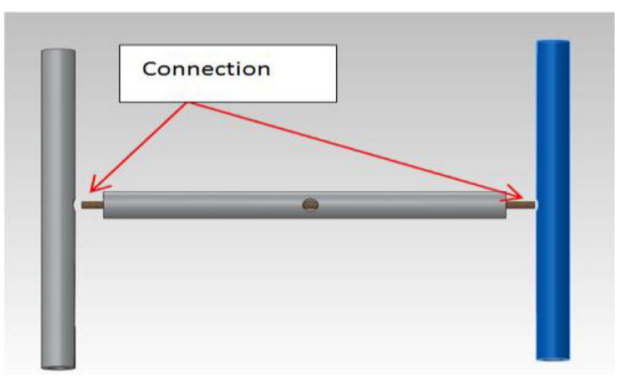

Figure 10. Bungee connection example.

To reduce the damage due to crash, the bicycle frame and two wheels are independently connected to the support motion platform using magnets. Figure 11 shows a prototype of the surrogate bicycle.

\section{Driving System of the Surrogate Bicycle}

To mimic the real bicycle motion and fit the micro Doppler properties in the view of $77 \mathrm{GHz}$ automotive radar, a pedal motion driving system also was developed. The driving system consists of a motor, a protective box, a one-to-two gear set and two paddles crank arms (shown in Figure 12). The mannequin rider has passive joints in the hips and knees. The A rotary shaft is connected to each foot of the rider and magnetically connected to the crank arm of the bicycle. The speed of pedal motion is controlled by a battery operated controller through Zigbee wireless network. The motion controller and battery are hidden in the mannequin body. An acceleration sensor is integrated into the controller to detect crash event. The accelerometer can detect the shock caused by the crash and shut down the power immediately to protect the motor.

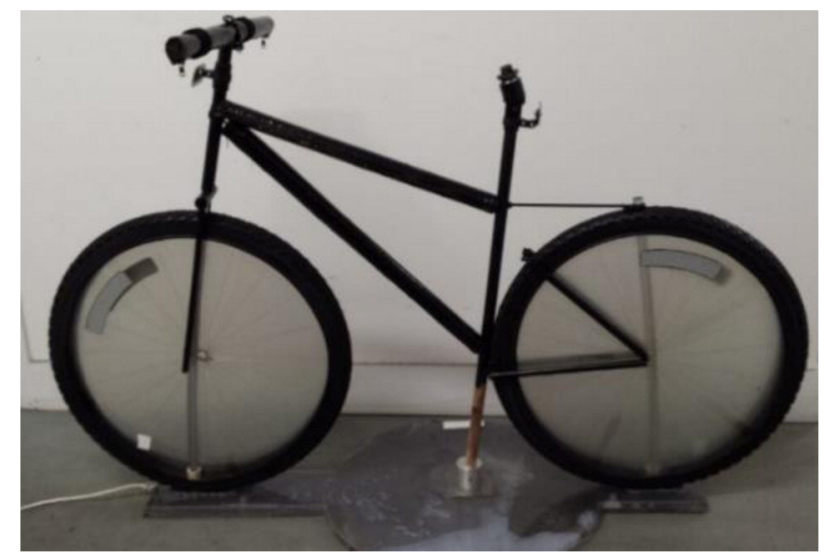

Figure 11. The prototype of surrogate bicycle.

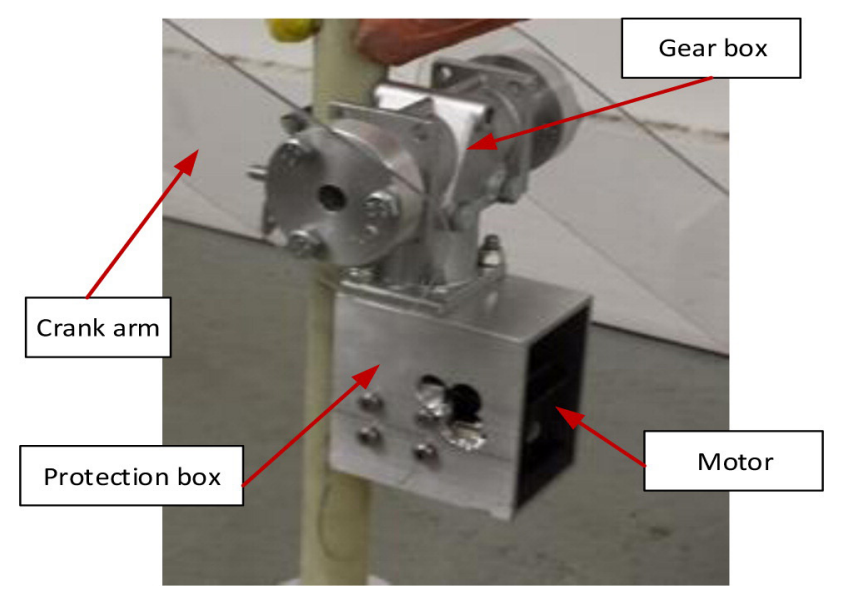

Figure 12. Pedal driving system.

\section{RCS Evaluation of the Surrogate Bicycle}

To compare the designed surrogate bicycle with the real bicycle, a comparative measurement of RCS of a real 26" bicycle and that of the surrogate bicycle was conducted. In addition to calibrated RCS level, the mean values of RCS level (in dBsm) within a 2-degree running window was calculated to reveal global pattern variation as a function of angle. The results are shown in Figure 13. The RCS measurements depicted by two plots (blue represents a surrogate bicycle, black represents a real bicycle) match each other in 360 degree very well. That means, from the view of the radar, TASI's surrogate bicycle can accurately represent the 26 " bicycle.

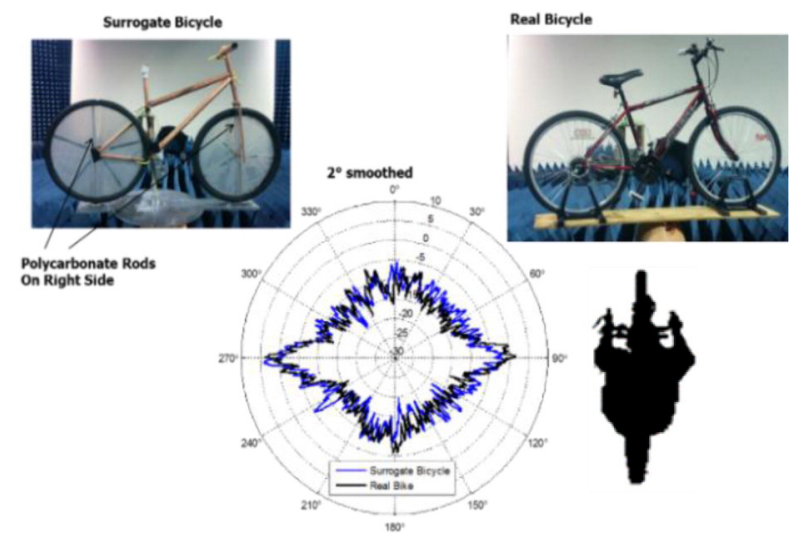

Figure 13. RCS comparison of the prototype and real bicycle. 


\section{Crash Test Results}

The final surrogate bicyclist rider weights $7 \mathrm{lbs}$. and the surrogate bicycle weights $15 \mathrm{lbs}$. The center of gravity of the whole surrogate bicyclist is below the top of the wheels. Hundreds of test runs were conducted to evaluate the BPCS test scenarios using the surrogate bicyclist described in this paper. The crash survivability of the surrogate bicyclist was also conducted using a pickup truck and passenger cars at different vehicle speed, different bicycle moving speed. The crash speeds range from 10 to $30 \mathrm{mph}$. The crash tests were performed to the side and rear of the surrogate bicyclist. Figure 14 shows several crash scenarios.

The bicyclist performed very well in the crash tests. The surrogate bicyclist can always be reassembled less than 5 minutes (not counting moving the bicyclist back to original position after traveling 10-15 $\mathrm{mph}$ for over 10 seconds). Damage to the bicycle rider was rare and all damages to the bicycle rider could be repaired quickly on site. The crash damage occurs more to the surrogate bicycle since the main crash force were on the frame and wheels. The minor damage to the surrogate bicycle at high speed crash could be quickly repaired on site. Severe damages sometimes occur when the bicyclist was run over by the vehicle and dragged by the vehicle wheels (especially in crash tests using a pick-up truck).

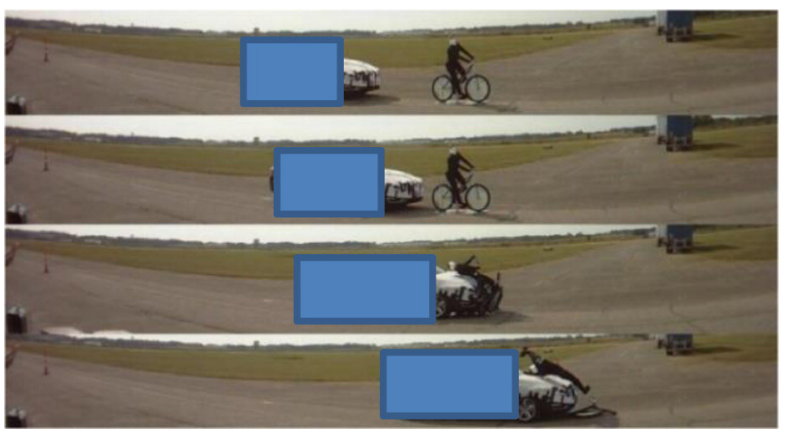

a. A stationary bicycle crashed in the rear by a vehicle with speed $20 \mathrm{mph}$.

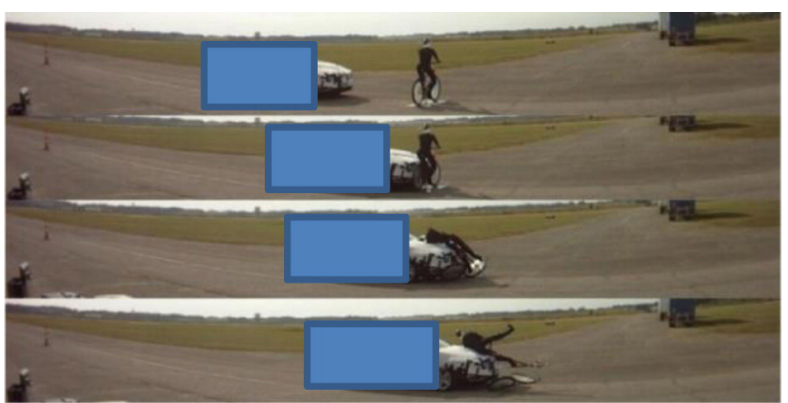

b. A stationary bicycle crashed on the left side by a vehicle with speed $20 \mathrm{mph}$.

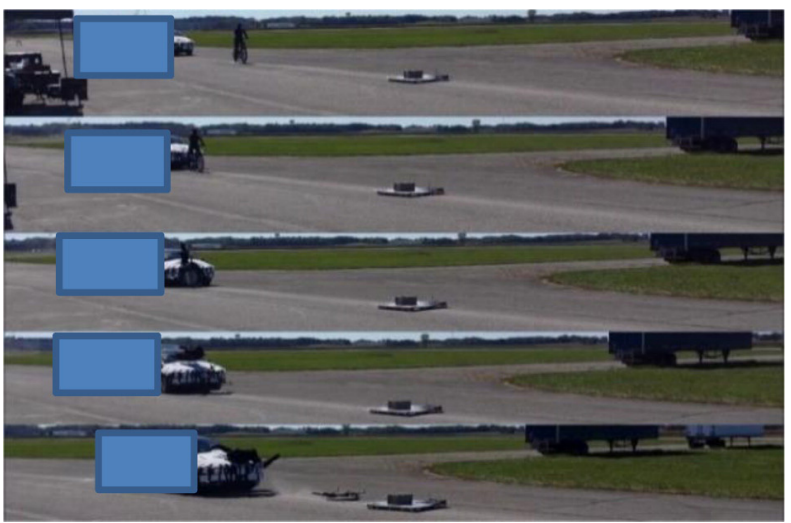

c. Bicycle along the road at speed $4.1 \mathrm{~m} / \mathrm{s}$, Vehicle with speed $40 \mathrm{mph}$, crashed from the rear.

\section{Discussion}

This paper summarized the current result of the development of the surrogate bicyclist for BPCS performance evaluation. Representing the US bicyclist population in the view of $77 \mathrm{GHz}$ automotive radar and camera, and crash survivability were the main focus of the study. The features were focused on the currently used bicyclist detection technology such as radar and camera. There was in depth study for each specification of the surrogate bicyclist, such as the size of the rider, the RCS of the bicycle, the pedaling speed, etc. The detailed study of these specific topics will be published later. Other aspects of the surrogate bicyclist such as visual clothing color, Lidar reflectivity, and facial features, are still under study and will be published. The surrogate bicyclist is also being improved for its crash survivability and the easiness to assemble.

\section{Conclusion}

The surrogate bicycle for performance evaluation of bicyclist precollision system was developed. The surrogate bicyclist was designed to represent the visual and radar characteristics of real bicycles and cyclists in the United States. The size of bicycle surrogate mimics the 26 inch adult bicycle, which is the most popular adult bicycle sold in the US. The RCS of the surrogate bicycle is wellrepresentative of the RCS of a real 26" bicycle from all 360 degree angles. Hundreds of crash tests with different motion scenarios were conducted. The results showed that it performed very well.

\section{References}

1. "Traffic Safety Facts: Bicyclists and Other Cyclists," National Highway Traffic Safety Administration, accessed January 14, 2016, http://www-nrd.nhtsa.dot.gov/Pubs/812151.pdf.

2. Aoki, H., Aga, M., Miichi, Y., Matsuo, Y. et al., "Safety Impact Methodology (SIM) for Effectiveness Estimation of a PreCollision System (PCS) by Utilizing Driving Simulator Test and EDR Data Analysis," SAE Technical Paper 2010-01-1003, 2010, doi:10.4271/2010-01-1003.

3. Gandhi T. and Trivedi M. M., "Pedestrian Protection Systems: Issues, Survey, and Challenges," Intelligent Transportation Systems, IEEE Transactions on, vol. 8, pp. 413-430, 2007-01-01 2007.

4. Li, T. Cao X. and Xu Y., "An effective crossing cyclist detection on a moving vehicle," in Intelligent Control and Automation (WCICA), 2010 8th World Congress on, 2010, pp. 368-372.

5. Kruse F., Fölster F., Ahrholdt M., Rohling H., Meinecke M., and To T., "Target classification based on near-distance radar sensors," in Intelligent Vehicles Symposium, 2004 IEEE, 2004, pp. 722-727.

6. Meinecke M., Obojski M. A., Töns M., Doerfler R., MarchalP., Letellier L., Gavrila D., and Morris R., "Approach for protection of vulnerable road users using sensor fusion techniques," in International Radar Symposium, 2003, pp. 125-130.

7. "Bicycling and walking in the United States, 2014 benchmarking report," Alliance for Biking \& Walking, accessed January 14, 2016, https://www.bikewalkalliance.org/storage/ documents/reports/2014BenchmarkingReport.pdf 
8. Qiang Y., Chien S., Brink J., Yaobin C., Linxi L., Chi-Chih C., Good D., and Sherony R., "Mannequin development for pedestrian pre-Collision System evaluation," in Intelligent Transportation Systems (ITSC), 2014 IEEE 17th International Conference on, 2014, pp. 1626-1631.

\section{Contact Information}

Drs. Qiang Yi and Stanley Chien

Transportation Active Safety Institute, Indiana University Purdue University Indianapolis

723 W. Michigan St., SL160, Indianapolis, IN 46202

yiq@iupui.edu

schien@iupui.edu

Phone: (317) 274-2760

\section{Acknowledgments}

This project was sponsored by Toyota's Collaborative Safety Research Center.

\section{Definitions/Abbreviations}

BPCS - Bicyclist Pre-Collision System

AEB - Automatic Emergency Braking

CIB - Collision Imminent Breaking

RCS - Radar Cross Section

FARS - Fatality Analysis Reporting System

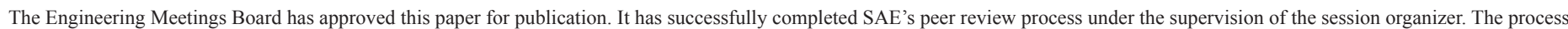
requires a minimum of three (3) reviews by industry experts.

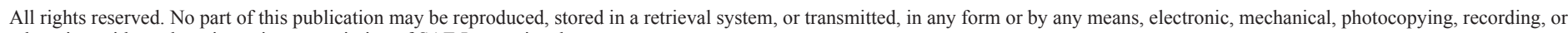
otherwise, without the prior written permission of SAE International.

Positions and opinions advanced in this paper are those of the author(s) and not necessarily those of SAE International. The author is solely responsible for the content of the paper.

ISSN 0148-7191

http://papers.sae.org/2016-01-1447 